doi:10.13108/2019-11-1-19

UDC 517.5

\title{
ON AN INTERPOLATION PROBLEM IN THE CLASS OF FUNCTIONS OF EXPONENTIAL TYPE IN A HALF-PLANE
}

\author{
B.V. VYNNYTS'KYI, V.L. SHARAN, I.B. SHEPAROVYCH
}

Abstract. Solvability conditions for interpolation problem $f(n)=d_{n}, \quad n \in \mathbb{N}$ in the class of entire functions satisfying the condition $|f(z)| \leqslant e^{\pi|\operatorname{Im} z|+o(|z|)}, z \rightarrow \infty$ are well known. In the presented paper we study the interpolation problem $f\left(\lambda_{n}\right)=d_{n}$ in the class of exponential type functions in the half-plane. We find sufficient solvability conditions for the considerate problem. In particular, a sufficient part of Carleson's interpolation theorem is generalized and an analogue of a classic interpolation condition is found in the form

$$
\sum_{j=k}^{\infty} \operatorname{Re}\left(-\xi_{j} \frac{\lambda_{k}^{2}-1}{\lambda_{k}+\overline{\lambda_{j}}}\right) \leqslant c_{3}, \quad \xi_{j}:=\frac{\operatorname{Re} \lambda_{j}}{1+\left|\lambda_{j}\right|^{2}} .
$$

The necessity of sufficient conditions is also discussed. The results are applied to studying a problem on splitting and searching an analogue of the identity $2 \cos z=\exp (-i z)+\exp (i z)$ for each function of exponential type in the half-plane. We prove that each holomorphic in the right-hand half-plane function $f$ obeying the , estimate $|f(z)| \leqslant O(\exp (\sigma|\operatorname{Im} z|))$ can be represented in the form $f=f_{1}+f_{2}$ and the functions $f_{1}$ and $f_{2}$ holomorphic in the right-hand half-plane satisfy conditions

$$
\left|f_{1}(z)\right| \leqslant O\left(\exp \left(|z| h_{-}(\varphi)\right)\right) \text { and }\left|f_{2}(z)\right| \leqslant O\left(\exp \left(|z| h_{+}(\varphi)\right)\right),
$$

where $\sigma \in[0 ;+\infty), z=r e^{i \varphi}$,

$$
h_{+}(\varphi)=\left\{\begin{array}{ll}
\sigma|\sin \varphi|, & \varphi \in\left[0 ; \frac{\pi}{2}\right], \\
0, & \varphi \in\left[-\frac{\pi}{2} ; 0\right],
\end{array} \quad h_{-}(\varphi)= \begin{cases}0, & \varphi \in\left[0 ; \frac{\pi}{2}\right], \\
\sigma|\sin \varphi|, & \varphi \in\left[-\frac{\pi}{2} ; 0\right] .\end{cases}\right.
$$

The paper uses methods works by L. Carleson, P. Jones, K. Kazaryan, K. Malyutin and other mathematicians.

Keywords: holomorphic functions of exponential type in the half-plane, interpolation, splitting of holomorphic functions

Mathematics Subject Classifcation: 30E05, 30D15

\section{INTRODUCTION} [1]

It is known that for each sequence $d=\left(d_{n}\right) \in l^{\infty}$ there exists an entire function $f$ such that

$$
\begin{aligned}
& f(n)=d_{n}, \quad n \in \mathbb{N}, \\
& |f(z)| \leqslant e^{\pi|\operatorname{Im} z|+o(|z|)}, \quad z \rightarrow \infty .
\end{aligned}
$$

In (1.2), "o(|z|)" can not be omitted [1], [2]. Our aim is to prove the following statement.

B.V. Vynnyts'kyi, V.L. Sharan, I.B. Sheparovych, On an interpolation problem in the Class OF FUNCTIONS OF EXPONENTIAL TYPE IN A HALF-PLANE.

(c)Vynnyts'kyi B.V., Sharan V.L., Sheparovych I.B. 2019.

Submitted June 1, $201 \%$. 
Theorem 1. For each sequence $\left(d_{n}\right) \in l^{\infty}$ there exists a holomorphic in the half-plane $\mathbb{C}_{+}=$ $\{z: \operatorname{Re} z>0\}$ function $f$ such that (1.1) holds and

$$
|f(z)| \leqslant c_{1} e^{\pi|\operatorname{Im} z|}, \quad z \in \mathbb{C}_{+} .
$$

Hereinafter $c_{j}$ stand for positive constants.

We let $h \in C[-\pi / 2 ; \pi / 2], \sigma \in[0 ;+\infty), h_{0}(\varphi)=\sigma|\sin \varphi|$,

$$
h_{+}(\varphi)=\left\{\begin{array}{ll}
\sigma|\sin \varphi|, & \varphi \in\left[0 ; \frac{\pi}{2}\right], \\
0, & \varphi \in\left[-\frac{\pi}{2} ; 0\right],
\end{array} \quad h_{-}(\varphi)= \begin{cases}0, & \varphi \in\left[0 ; \frac{\pi}{2}\right], \\
\sigma|\sin \varphi|, & \varphi \in\left[-\frac{\pi}{2} ; 0\right] .\end{cases}\right.
$$

and let $H^{\infty}\left(\mathbb{C}_{+} ; h\right)$ be the space of functions $f$ holomorphic in $\mathbb{C}_{+}$obeying

$$
\|f\|:=\sup \left\{|f(z)| e^{-r h(\varphi)}: z=x+i y=r e^{i \varphi} \in \mathbb{C}_{+}\right\}<+\infty .
$$

We employ Theorem 1 and its modifications for proving the following statement.

Theorem 2. Let $\sigma \in[0 ;+\infty)$. Then each function $f \in H^{\infty}\left(\mathbb{C}_{+} ; h_{0}\right)$ is represented as

$$
f=f_{1}+f_{2}, \quad f_{1} \in H^{\infty}\left(\mathbb{C}_{+} ; h_{-}\right), \quad f_{2} \in H^{\infty}\left(\mathbb{C}_{+} ; h_{+}\right) .
$$

The problem on splitting (1.4), which is an analogue of the identity $\cos \sigma z=\frac{1}{2} e^{i \sigma z}+\frac{1}{2} e^{-i \sigma z}$, arises in seeking analogue of Paley-Wiener theorem for some weighted spaces and studying some convolution type equations (see [3, 4]). It was studied in works by V.M. Dilnyi [5, 6]. However, positive resolving is known mostly for spaces defined by $L_{2}$-metric. For the space $H^{\infty}\left(\mathbb{C}_{+} ; h_{0}\right)$, the issue remained open. Theorem 2 positively resolves this. A more complicated and important similar question for the space of exponential type in the half-plane defined by $L_{1}$-metric remains open.

Let $\lambda=\left(\lambda_{n}\right)=\left(\left|\lambda_{n}\right| e^{i \varphi_{n}}\right)$ be an arbitrary sequence of different complex numbers in the complex half-plane $\mathbb{C}_{+}, l^{\infty}(h ; \lambda)$ be the space of sequences $d$, for which

$$
\|d\|:=\sup \left\{\left|d_{n}\right| e^{-\left|\lambda_{n}\right| h\left(\varphi_{n}\right)}: n \in \mathbb{N}\right\}<+\infty .
$$

Let

$$
S(r):=\sum_{1<\left|\lambda_{k}\right| \leqslant r}\left(\frac{1}{\left|\lambda_{k}\right|^{2}}-\frac{1}{r^{2}}\right) \operatorname{Re} \lambda_{k} .
$$

Various interpolation problems in the classes of functions holomorphic in the half-plane were considered in many works, see [7-9] and the references therein. However, the solvability criterions of the interpolation problem

$$
f\left(\lambda_{n}\right)=d_{n}, \quad n \in \mathbb{N},
$$

in the class $H^{\infty}\left(\mathbb{C}_{+} ; h_{0}\right)$ is not known.

We employ some ideas from [7-9] and obtain the above formulated theorems on the base of the following statement, which in fact contains a sufficient part of the interpolation Carleson theorem; its elementary proof for the half-plane was provided, for instance, in [9]. 
Theorem 3. Let $\left(\lambda_{k}\right)$ be a sequence of different complex numbers in the half-plane $\mathbb{C}_{+}=$ $\{z: \operatorname{Re} z>0\}$ such that

$$
\begin{aligned}
& \sum_{\left|\lambda_{k}\right| \leqslant 1} \operatorname{Re} \lambda_{k}<+\infty \\
& \sup \left\{S(r)-\frac{\sigma}{\pi} \ln r: r \in[1 ;+\infty)\right\}<+\infty, \\
& \sum_{j=k}^{\infty} \operatorname{Re}\left(-\xi_{j} \frac{\lambda_{k}^{2}-1}{\lambda_{k}+\overline{\lambda_{j}}}\right) \leqslant c_{3}, \quad \xi_{j}:=\frac{\operatorname{Re} \lambda_{j}}{1+\left|\lambda_{j}\right|^{2}} .
\end{aligned}
$$

Moreover, let the sequence $\left(\lambda_{k}\right)$ is a subsequence of zeroes of a holomorphic in $\mathbb{C}_{+}$function $\Omega$ such that

$$
\left|\frac{\Omega(z)\left(z+\overline{\lambda_{k}}\right)}{\left(z-\lambda_{k}\right) \operatorname{Re} \lambda_{k} \Omega^{\prime}\left(\lambda_{k}\right)}\right| \leqslant c_{0} e^{r h_{0}(\varphi)} e^{-\left|\lambda_{k}\right| h_{0}\left(\varphi_{n}\right)}, \quad z=x+i y=r e^{i \varphi} \in \mathbb{C}_{+}, \quad k \in \mathbb{N} .
$$

Then for each sequence $d \in l^{\infty}\left(h_{0} ; \lambda\right)$ there exists a function $f \in H^{\infty}\left(\mathbb{C}_{+} ; h_{0}\right)$ satisfying condition (1.5).

Remark 1. If $\sigma=0$, then conditions (1.6) and (1.7) are equivalent to the condition

$$
\sum_{j=1}^{\infty} \frac{\operatorname{Re} \lambda_{j}}{1+\left|\lambda_{j}\right|^{2}}<+\infty
$$

and if $\Omega(z)=B(z)$ is the Blaschke product for $\mathbb{C}_{+}$, then condition (1.9) is equivalent to the Carleson condition

$$
\inf \left\{\left|\prod_{k=1, k \neq n}^{\infty} \frac{\lambda_{n}-\lambda_{k}}{\lambda_{n}+\overline{\lambda_{k}}}\right|: n \in \mathbb{N}\right\} \geqslant \delta>0,
$$

while the latter implies (1.8), see, for instance, [9]. The issue on necessity of conditions (1.8) and (1.9) remains for us open. Some comments on this issues are given in the end of the paper.

\section{Proof of Theorem 3}

Let $s_{0}(t)=\sum_{1<\left|\lambda_{k}\right| \leqslant t} \operatorname{Re} \lambda_{k}$. Since

$$
\frac{1}{t^{2}} \leqslant \frac{4}{3}\left(\frac{1}{t^{2}}-\frac{1}{4 s^{2}}\right) \quad \text { as } \quad|t| \leqslant|s|
$$

then

$$
\begin{aligned}
s_{0}(r) & \leqslant r^{2} \sum_{1<\left|\lambda_{k}\right| \leqslant r} \frac{\operatorname{Re} \lambda_{k}}{\left|\lambda_{k}\right|^{2}} \leqslant r^{2} \frac{4}{3} \sum_{1<\left|\lambda_{k}\right| \leqslant r}\left(\frac{1}{\left|\lambda_{k}\right|^{2}}-\frac{1}{(2 r)^{2}}\right) \operatorname{Re} \lambda_{k} \\
& \leqslant r^{2} \frac{4}{3} \sum_{1<\left|\lambda_{k}\right| \leqslant 2 r}\left(\frac{1}{\left|\lambda_{k}\right|^{2}}-\frac{1}{(2 r)^{2}}\right) \operatorname{Re} \lambda_{k}=\frac{4}{3} r^{2} S(2 r) .
\end{aligned}
$$

This is why conditions (1.6) and (1.7) implies the convergence of the series $\sum_{j=1}^{\infty}\left(\frac{\operatorname{Re} \lambda_{j}}{1+\left|\lambda_{j}\right|^{2}}\right)^{2}$ and $\sum_{j=1}^{\infty} \operatorname{Re} \lambda_{j}\left(1+\left|\lambda_{j}\right|^{2}\right)^{-3 / 2}$. Therefore, $\xi_{j} \rightarrow 0$. This is why, as in [7-9], in the proof of Theorem 3 
we can assume that the sequence $\left(\xi_{j}\right)$ is non-increasing. Let

$$
\Psi_{j}(z)=-\xi_{j} \frac{z^{2}-1}{z+\overline{\lambda_{j}}} \quad \text { and } \quad F_{k}(z)=\exp \left(-\sum_{j=k}^{\infty} \Psi_{j}(z)\right) .
$$

The latter series converges uniformly on compact sets in $\mathbb{C}_{+}$. Let us show that the sought function is

$$
f(z)=\sum_{k=1}^{\infty} d_{k} \frac{\Omega(z)\left(z+\overline{\lambda_{k}}\right)}{\left(z-\lambda_{k}\right) \Omega^{\prime}\left(\lambda_{k}\right) 2 \operatorname{Re} \lambda_{k}}\left(\frac{1+z}{1+\lambda_{k}}\right)^{2}\left(\frac{2 \operatorname{Re} \lambda_{k}}{z+\bar{\lambda}_{k}}\right)^{2} \frac{e^{\xi_{k} \lambda_{k}}}{e^{\xi_{k} z}} \frac{F_{k}(z)}{F_{k}\left(\lambda_{k}\right)} .
$$

Indeed,

$$
\frac{z^{2}-1}{z+\overline{\lambda_{j}}}=z-\frac{1+z \overline{\lambda_{j}}}{z+\overline{\lambda_{j}}}, \quad \operatorname{Re} \frac{1+z \overline{\lambda_{j}}}{z+\overline{\lambda_{j}}}=\frac{\left(1+|z|^{2}\right) \operatorname{Re} \lambda_{j}+\left(1+\left|\lambda_{j}\right|^{2}\right) \operatorname{Re} z}{\left|z+\overline{\lambda_{j}}\right|^{2}}
$$

and

Hence,

$$
\operatorname{Re} \Psi_{j}(z)=\frac{\left(1+|z|^{2}\right) \operatorname{Re}^{2} \lambda_{j}}{\left(1+\left|\lambda_{j}\right|^{2}\right)\left|z+\overline{\lambda_{j}}\right|^{2}}+\frac{\operatorname{Re} \lambda_{j} \operatorname{Re} z}{\left|z+\overline{\lambda_{j}}\right|^{2}}-\xi_{j} \operatorname{Re} z
$$

Moreover, see [9],

$$
\begin{aligned}
\left|F_{k}(z)\right| & \leqslant \exp \left(\sum_{j=k}^{\infty}\left(-\frac{\left(1+|z|^{2}\right) \operatorname{Re}^{2} \lambda_{j}}{\left(1+\left|\lambda_{j}\right|^{2}\right)\left|z+\overline{\lambda_{j}}\right|^{2}}+\xi_{j} \operatorname{Re} z\right)\right) \\
& \leqslant \exp \left(\xi_{k} \operatorname{Re} z\right) \exp \left(\sum_{j=k}^{\infty}\left(-\frac{\left(1+|z|^{2}\right) \operatorname{Re}^{2} \lambda_{j}}{\left(1+\left|\lambda_{j}\right|^{2}\right)\left|z+\overline{\lambda_{j}}\right|^{2}}\right)\right) .
\end{aligned}
$$

$$
\begin{aligned}
\left|\frac{2 \operatorname{Re} \lambda_{j}}{1+\lambda_{j}} \frac{z+1}{z+\overline{\lambda_{j}}}\right|^{2} & \leqslant 4 \frac{\operatorname{Re} \lambda_{j}}{\left(1+\left|\lambda_{j}\right|^{2}\right)\left|z+\overline{\lambda_{j}}\right|^{2}}\left(\left(|z|^{2}+1\right) \operatorname{Re} \lambda_{j}+\left(1+\left|\lambda_{j}\right|^{2}\right) \operatorname{Re} z\right) \\
& =4 \operatorname{Re} \frac{\operatorname{Re} \lambda_{j}}{1+\left|\lambda_{j}\right|^{2}} \frac{1+z \overline{\lambda_{j}}}{z+\overline{\lambda_{j}}}
\end{aligned}
$$

In addition, according condition (1.8),

$$
\left|F_{k}\left(\lambda_{k}\right)\right|=\exp \left(-\sum_{j=k}^{\infty} \operatorname{Re}\left(-\xi_{j} \frac{\lambda_{k}^{2}}{\lambda_{k}+\overline{\lambda_{j}}}+\xi_{j} \frac{1}{\lambda_{k}+\overline{\lambda_{j}}}\right)\right) \geqslant c_{2} .
$$

Therefore,

$$
\begin{aligned}
\mid d_{k} \frac{\Omega(z)\left(z+\overline{\lambda_{k}}\right)}{\left(z-\lambda_{k}\right) \Omega^{\prime}\left(\lambda_{k}\right) 2 \operatorname{Re} \lambda_{k}} & \left(\frac{1+z}{1+\lambda_{k}}\right)^{2}\left(\frac{2 \operatorname{Re} \lambda_{k}}{z+\bar{\lambda}_{k}}\right)^{2} \frac{e^{\xi_{k} \lambda_{k}}}{e^{\xi_{k} z}} \frac{F_{k}(z)}{F_{k}\left(\lambda_{k}\right)} \mid \\
& \leqslant c_{3}\left|\left(\frac{1+z}{1+\lambda_{k}}\right)^{2}\left(\frac{2 \operatorname{Re} \lambda_{k}}{z+\bar{\lambda}_{k}}\right)^{2} \frac{e^{\xi_{k} \lambda_{k}}}{e^{\xi_{k} z}} \frac{F_{k}(z)}{F_{k}\left(\lambda_{k}\right)}\right| \\
& \leqslant c_{4} \frac{\operatorname{Re} \lambda_{k}}{1+\left|\lambda_{k}\right|^{2}} \operatorname{Re} \frac{1+z \overline{\lambda_{k}}}{z+\overline{\lambda_{k}}} \exp \left(-\sum_{j \geqslant k}\left(\frac{\operatorname{Re} \lambda_{k}}{1+\left|\lambda_{k}\right|^{2}} \operatorname{Re} \frac{1+z \overline{\lambda_{k}}}{z+\overline{\lambda_{k}}}\right)\right) .
\end{aligned}
$$

Since

$$
\sum_{k=1}^{\infty}\left|a_{k}\right| \exp \left(-\sum_{j=k}^{\infty}\left|a_{j}\right|\right)<1
$$

we arrive at the statement of Theorem 3 . 


\section{Proof of TheOrem 1}

Lemma 3.1. Let $\sigma \in[0 ;+\infty)$, the function $\Omega \in H^{\infty}\left(\mathbb{C}_{+} ; h_{0}\right)$ has the zeroes at the points $\lambda_{k} \in \mathbb{C}_{+}$,

$$
\tilde{\Omega}_{k}(z)=\frac{\Omega(z)\left(z+\overline{\lambda_{k}}\right)}{z-\lambda_{k}}, \quad \tau_{k}=\frac{\delta_{k}}{1+\sqrt{1+\delta_{k}^{2}}},
$$

where $\delta_{k}=1$ if $\operatorname{Re} \lambda_{k}<1$ or if $\sigma=0$, and $\delta_{k}=\left(\operatorname{Re} \lambda_{k}\right)^{-1}$ if $\sigma>0$ and $\operatorname{Re} \lambda_{k} \geqslant 1$. Then

$$
\left|\tilde{\Omega}_{k}(z)\right| \leqslant \frac{c_{2}}{\tau_{k}} \exp (\sigma|y|)
$$

as $z \in \mathbb{C}_{+}, k \in \mathbb{N}$.

Proof. Since $\tau_{k} \in(0 ; 1)$ and $\delta_{k}=\frac{2 \tau_{k}}{1-\tau_{k}^{2}}$, the circles

$$
\begin{aligned}
U_{k} & :=\left\{\varsigma \in \mathbb{C}:\left|\frac{\varsigma-\lambda_{k}}{\varsigma+\overline{\lambda_{k}}}\right|<\tau_{k}\right\} \\
& =\left\{\varsigma=\xi+i \eta \in \mathbb{C}:\left(\xi-\frac{1+\tau_{k}^{2}}{1-\tau_{k}^{2}} \operatorname{Re} \lambda_{k}\right)^{2}+\left(\eta-\operatorname{Im} \lambda_{k}\right)^{2}<\left(\frac{2 \tau_{k} \operatorname{Re} \lambda_{k}}{1-\tau_{k}^{2}}\right)^{2}\right\}
\end{aligned}
$$

are contained in $\mathbb{C}_{+}$. Then

$$
\left|\tilde{\Omega}_{k}(z)\right| \leqslant \frac{|\Omega(z)|}{\tau_{k}} \leqslant \frac{c_{1}}{\tau_{k}} \exp (\sigma|y|) \quad \text { if } \quad\left|\frac{z-\lambda_{k}}{z+\overline{\lambda_{k}}}\right| \geqslant \tau_{k} .
$$

If $\left|\frac{z-\lambda_{k}}{z+\lambda_{k}}\right|<\tau_{k}$, then by the maximum principle we obtain

$$
\left|\tilde{\Omega}_{k}(z)\right| \leqslant \max \left\{\frac{c_{1} e^{\sigma|\operatorname{Im} \varsigma|}}{\tau_{k}}:\left|\frac{\varsigma-\lambda_{k}}{\varsigma+\overline{\lambda_{k}}}\right|=\tau_{k}\right\} \leqslant \frac{1}{\tau_{k}} e^{\sigma|y|+2 \sigma \delta_{k} \operatorname{Re} \lambda_{k}} .
$$

Since $\sigma \delta_{k} \operatorname{Re} \lambda_{k} \leqslant \sigma$, this completes the proof.

We note that

$$
\tau_{k} \geqslant \frac{1}{3} \operatorname{Re} \lambda_{k}
$$

if $\sigma>0$ and $\operatorname{Re} \lambda_{k} \geqslant 1$. Therefore, the proven lemma implies that the sequence $\lambda=(k)$ satisfies all assumptions of Theorem 3 for $\sigma=\pi$, and at that, we can take $\Omega(z)=\sin \pi z$. In addition, $l^{\infty} \subset l^{\infty}\left(h_{0} ; \lambda\right)$ if $\lambda=(k)$. This is why Theorem 1 follows Theorem 3.

\section{Proof of Theorem 2}

Lemma 4.1. Let $\left(\lambda_{k}\right)$ be a sequence of different complex numbers in the half-plane $\mathbb{C}_{+}=$ $\{z: \operatorname{Re} z>0\}$ such that inequalities (1.6), (1.8) hold and

$$
\sup \left\{S(r)-\frac{\sigma}{2 \pi} \ln r: r \in[1 ;+\infty)\right\}<+\infty .
$$

Let also $\left(\lambda_{k}\right)$ be a subsequence of zeroes of a holomorphic in $\mathbb{C}_{+}$function $\Omega$ such that

$$
\left|\frac{\Omega(z)\left(z+\overline{\lambda_{k}}\right)}{\left(z-\lambda_{k}\right) \operatorname{Re} \lambda_{k} \Omega^{\prime}\left(\lambda_{k}\right)}\right| \leqslant c_{0} e^{r h_{-}(\varphi)} e^{-\left|\lambda_{k}\right| h_{-}\left(\varphi_{n}\right)}, \quad z=x+i y=r e^{i \varphi} \in \mathbb{C}_{+}, \quad k \in \mathbb{N} .
$$

Then for each sequence $\left(d_{k}\right) \in l^{\infty}\left(h_{+} ; \lambda\right)$ there exists a holomorphic in $\mathbb{C}_{+}$function $f \in$ $H^{\infty}\left(\mathbb{C}_{+} ; h_{+}\right)$satisfying condition (1.5).

The proof of this lemma reproduces literally the proof of Theorem 3. 
Lemma 4.2. Let $\sigma \in[0 ;+\infty)$, a function $\Omega \in H^{\infty}\left(\mathbb{C}_{+} ; h_{+}\right)$has zeroes at the points $\lambda_{k} \in \mathbb{C}_{+}$ and

Then

$$
\tilde{\Omega}_{k}(z)=\frac{\Omega(z)\left(z+\overline{\lambda_{k}}\right)}{z-\lambda_{k}} .
$$

$$
\left|\tilde{\Omega}_{k}(z)\right| \leqslant \frac{c_{2}}{\tau_{k}} \exp \left(r h_{+}(\varphi)\right) \quad \text { as } \quad z=x+i y=r e^{i \varphi} \in \mathbb{C}_{+} .
$$

The proof of this lemma is similar to the proof of Lemma 3.1.

We proceed to proving Theorem 2 . We assume that $\sigma=\pi$. Let $\Omega(z)=e^{-i \frac{\pi}{2}(z-1)} \sin \frac{\pi}{2}(z-1)$. This functions has zeroes in $\mathbb{C}_{+}$at the points $\lambda_{k}=2 k-1, k \in \mathbb{N}$, and $\Omega \in H^{\infty}\left(\mathbb{C}_{+} ; h_{+}\right)$. At that, $\left|\Omega^{\prime}\left(\lambda_{k}\right)\right|=\pi / 2$, and according Lemma 4.2 , the sequence $\lambda_{k}=2 k-1$ satisfies all assumptions of Lemma 4.1. Let $d_{k}=f\left(\lambda_{k}\right)$. Then $\left(d_{k}\right) \in l^{\infty}\left(h_{+} ; \lambda\right)$. Hence, according Lemma 4.1, there exists a function $f_{0} \in H^{\infty}\left(\mathbb{C}_{+} ; h_{+}\right)$such that $f_{0}\left(\lambda_{k}\right)=f\left(\lambda_{k}\right), k \in \mathbb{N}$. Let $\tilde{f}(z)=\frac{f(z)-f_{0}(z)}{\Omega(z)}$. Since [10]

$$
\left|\sin \frac{\pi}{2}(z-1)\right| \geqslant c_{0} \exp \left(\frac{\pi}{2}|\operatorname{Im} z|\right)
$$

outside the circles $\left|z-\lambda_{k}\right| \leqslant \varepsilon$ and therefore, outside these circles the estimate

$$
|\tilde{f}(z)| \leqslant c_{5} \exp \left(r h_{-}(\varphi)\right), \quad z=x+i y=r e^{i \varphi},
$$

holds true. Now by the maximum principle we infer that $\tilde{f} \in H^{\infty}\left(\mathbb{C}_{+} ; h_{-}\right)$. Moreover,

$$
f(z)=\tilde{f}(z) \Omega(z)+f_{0}(z)=\frac{1}{2 i} \tilde{f}(z)+f_{0}(z)-\frac{1}{2 i} e^{-i \pi z} \tilde{f}(z) .
$$

Since $f_{1}(z):=\frac{1}{2 i} \tilde{f}(z) \in H^{\infty}\left(\mathbb{C}_{+} ; h_{-}\right)$and $f_{2}(z):=f_{0}(z)-\frac{1}{2 i} e^{-i \pi z} \tilde{f}(z) \in H^{\infty}\left(\mathbb{C}_{+} ; h_{+}\right)$, this completes the proof of Theorem 2 .

\section{ADDENDA AND REMARKS}

Conditions (1.6) and (1.7) are necessary for the statement of Theorem 3. Indeed, let $Q(z)=$ $f(z) \frac{z-\lambda_{1}}{z+\lambda_{1}}$, where $f \in H^{\infty}\left(\mathbb{C}_{+} ; h_{0}\right)$ is a function such that $f\left(\lambda_{1}\right)=1$ and $f\left(\lambda_{k}\right)=0$ if $k \neq 1$. Then $Q \in H^{\infty}\left(\mathbb{C}_{+} ; h_{0}\right)$ and $\left(\lambda_{k}\right)$ is a sequence of zeroes of the function $Q$. This is why, by the generalized Carleman formula [11] we obtain (1.6) and (1.7) [12]. If the sequence $\left(\lambda_{k}\right)$ satisfies conditions (1.6) and (1.7), then [10] there exists a function $f \in H^{\infty}\left(\mathbb{C}_{+} ; h_{0}\right)$, for which this is a sequence of its zeroes. Each function $f \in H^{\infty}\left(\mathbb{C}_{+} ; h_{0}\right), f \neq 0$, is represented as [11]

$$
f(z)=e^{i a_{0}+a_{1} z} \tilde{B}(z) \tilde{T}(z),
$$

where $a_{0} \in \mathbb{R}$ and $a_{1} \in \mathbb{R}$ are constants,

$$
\begin{gathered}
Q_{1}(t ; z)=\frac{(t z+i)^{2}}{\left(1+t^{2}\right)^{2}(t+i z)}, \\
\tilde{T}(z)=\exp \left\{\frac{1}{\pi i} \int_{-\infty}^{+\infty} Q_{1}(t ; z)\left(\ln \left|f_{0}(i t)\right| d t+d h(t)\right)\right\}, \quad \tilde{B}(z)=\prod_{j=1}^{\infty} W_{j}(z),
\end{gathered}
$$

$f_{0}(i t)=f(i t)$ are angular boundary values of $f(z)$ on $\partial \mathbb{C}_{+}, h(t)$ is a non-increasing function (a singular boundary function of the function $f$ ), whose derivative vanishes everywhere,

$$
W_{j}(z)=\frac{z-\lambda_{j}}{z+\overline{\lambda_{j}}} \quad \text { as } \quad\left|\lambda_{j}\right| \leqslant 1, \quad W_{j}(z)=\frac{1-\frac{z}{\lambda_{j}}}{1+\frac{z}{\bar{\lambda}_{j}}} \exp \left(\frac{z}{\lambda_{j}}+\frac{z}{\overline{\lambda_{j}}}\right) \quad \text { as } \quad\left|\lambda_{j}\right|>1 .
$$

In [13], the following statement was proved. 
Proposition 1. If $f \in H^{\infty}\left(\mathbb{C}_{+} ; h_{0}\right)$ and $f \not \equiv 0$, then $\left.\left.1_{a}\right) \log \left|f_{0}\right| \in L_{1}^{\text {loc }}(i \mathbb{R}), 2_{a}\right)$ $\left.f_{0}(i y) \exp (-\sigma|y|) \in L^{\infty}(\mathbb{R}), 1_{b}\right) \sup \{K(r): r \in[1 ;+\infty)\}<+\infty$, and (1.6) holds, where

$$
\begin{aligned}
K(r) & :=K_{Z}(r)+K_{S}(r)+K_{B}(r), \quad K_{Z}(r):=2 \sum_{1<\left|\lambda_{k}\right| \leqslant r}\left(\frac{1}{\left|\lambda_{k}\right|^{2}}-\frac{1}{r^{2}}\right) \operatorname{Re} \lambda_{k}, \\
K_{S}(r) & :=-\frac{1}{\pi} \int_{1 \leqslant|t| \leqslant r}\left(\frac{1}{|t|^{2}}-\frac{1}{r^{2}}\right) d h(t), \\
K_{B}(r) & :=-\frac{1}{\pi} \int_{1 \leqslant|t| \leqslant r}\left(\frac{1}{|t|^{2}}-\frac{1}{r^{2}}\right) \log \left|f_{0}(i t)\right| d t .
\end{aligned}
$$

Vice versa, if the sequence $\left(\lambda_{k}\right)$ of the points in the half-plane $\mathbb{C}_{+}$, a function $f_{0}: i \mathbb{R} \rightarrow \mathbb{C}$ and a non-increasing function $h: \mathbb{R} \rightarrow \mathbb{R}$, whose derivative vanishes almost everywhere are such that conditions $\left.\left.1_{a}\right), 2_{a}\right), 1_{b}$ ) and (1.6) hold, then the function $f$ defined by identity (5.1) is holomorphic in $\mathbb{C}_{+}$and satisfies the estimates $|f(z)| \leqslant c_{1} \exp \left(\sigma|y|+c_{1} x\right)$. At that, if in the product $\tilde{B}(z)$ we omit some of the factors, the above estimate remains true and the constant $c_{1}$ does not increase.

Employing this statement and some ideas from the proof of necessary part of Carleson intrerpolation theorem (see [14]), we confirm that each of the following conditions

$$
\begin{aligned}
& \prod_{j \in \mathbb{N}, j \neq k}\left|W_{j}\left(\lambda_{k}\right)\right| \geqslant c_{3} \exp \left(-c_{3} \operatorname{Re} \lambda_{k}\right), \quad k \in \mathbb{N}, \\
& \sum_{j \in \mathbb{N}, j \neq k}\left(\frac{2 \operatorname{Re} \lambda_{k} \operatorname{Re} \lambda_{j}}{\left|\lambda_{k}+\overline{\lambda_{j}}\right|^{2}}-\frac{2 \operatorname{Re} \lambda_{k} \operatorname{Re} \lambda_{j}}{1+\left|\lambda_{j}\right|^{2}}\right) \leqslant c_{4} \operatorname{Re} \lambda_{k}, \quad k \in \mathbb{N},
\end{aligned}
$$

is necessary for the solvability of interpolation problem $(1.5)$ in the class $H^{\infty}\left(\mathbb{C}_{+} ; h_{0}\right)$ for each sequence $d \in l^{\infty}\left(h_{0} ; \lambda\right)$. However, we fail in trying to prove the necessity of conditions (1.8) and (1.9). In view of this, it is useful to mention the inequality

$$
\begin{aligned}
\sum_{j=k}^{\infty} \operatorname{Re}\left(-\xi_{j} \frac{\lambda_{k}^{2}-1}{\lambda_{k}+\overline{\lambda_{j}}}\right) & =\sum_{j=k}^{\infty}\left(\xi_{j} \frac{\left(1+\left|\lambda_{k}\right|^{2}\right) \operatorname{Re} \lambda_{j}+\left(1+\left|\lambda_{j}\right|^{2}\right) \operatorname{Re} \lambda_{k}}{\left|\lambda_{k}+\overline{\lambda_{j}}\right|^{2}}-\xi_{j} \operatorname{Re} \lambda_{k}\right) \\
& \leqslant \sum_{j=k}^{\infty}\left(\frac{2 \operatorname{Re} \lambda_{k} \operatorname{Re} \lambda_{j}}{\left|\lambda_{k}+\overline{\lambda_{j}}\right|^{2}}-\frac{\operatorname{Re} \lambda_{k} \operatorname{Re} \lambda_{j}}{1+\left|\lambda_{j}\right|^{2}}\right) .
\end{aligned}
$$

\section{BIBLIOGRAPHY}

1. B. Levin. Lectures on entire functions. Amer. Math. Soc., Providence, RI (1996).

2. Y. Lyubarskii, K. Seip. Complete interpolating sequences for Paley-Wiener spaces and Muckenhoupt's (Ap) condition // Rev. Matem. Iberoamer. 13:2, 361-376 (1997).

3. B.V. Vynnytskyi. An extention of Paley-Wiener theorem // Matematychni Studii. 4, 37-44 (1995). (in Ukrainian.)

4. B.V. Vynnytskyi. On solutions of homogeneous convolution equation in one class of functions analytical in a semi-strip // Matematychni Studii. 7:.1, 41-52 (1997). (in Ukrainian.)

5. V.N. Dilnyi. Splitting of some spaces of analitic functions // Ufimskij Matem. Zhurn. 6:2, 26-35 (2014) [Ufa Math. J. 6:2, 26-35 (2014).]

6. B.V. Vynnytskyi, V.N. Dilnyi. On an analogue of Paley-Wiener's theorem for weighted Hardy spaces // Matematychni Studii. 14:1, 35-40 (2000). (in Ukrainian.) 
7. K. G. Malyutin. The problem of multiple interpolation in the half-plane in the class of analytic functions of finite order and normal type // Matem. Sborn. 184:2, 129-144 (1993). [Russian Acad. Sci. Sb. Math. 78:1, 253-266 (1994).]

8. K. G. Malyutin. Sets of regular growth of functions in a half-plane. II // Izv. RAN. Ser. Matem. 59:5, 103-126 (1995). [Izv. Math. 59:5, 983-1006 (1995).]

9. K.G. Kazaryan. Solution to a multipli interpolation problem in classes $H^{\infty}$ in the half-planje and strip // Izv. AN Arm. SSR. Matem. XXV:1, 66-82 (1990). (in Russian).

10. A.F. Leont'ev. Entire functions. Exponential series. Nauka, Moscow (1983). (in Russian).

11. N.V. Govorov. Riemann's boundary problem with infinite index. Nauka, Moscow (1986). [Operator Theory: Advances and Applications. 67. Birkhäuser, Basel. (1994).]

12. B.V. Vynnytskyi. On zeros of functions analytic in a half plane and completeness of systems of exponents // Ukr. Matem. Zhurn. 46:5, 484-500 (1994). [Urk. Math. J. 46:5, 514-532 (1994).]

13. B. Vynnytskyi, V. Sharan. On the factorization of one class of functions analytic in the halfplane // Matematychni Studii. 14:41-48 (2000).

14. K. Gofman. Banach spaces of analytic functions Inostr. Liter., Moscow (1963). (in Russian).

Bogdan Vasil'evich Vinnitskii,

Drohobych state pedagogical university named after Ivan Franko,

Stryiskaya str., 3

82100, Drohobych, Ukraine

E-mail: vynnytskyi@ukr.net

Vladimir Lukyanovich Sharan,

Drohobych state pedagogical university named after Ivan Franko,

Stryiskaya str., 3

82100, Drohobych, Ukraine

E-mail: volsharan@ukr.net

Irina Bogdanovna Sheparovich,

Drohobych state pedagogical university named after Ivan Franko,

Stryiskaya str., 3

82100, Drohobych, Ukraine

E-mail: isheparovych@ukr.net 